\title{
Use of a novel shorter minimum caliber needle for creating endoscopic tattoos for preoperative localization: a comparative ex vivo study $\square$
}

\section{다(1) $(9)$}

\author{
Authors \\ Kenichiro Imai ${ }^{1}$, Kinichi Hotta ${ }^{1}$, Sayo Ito ${ }^{1}$, Yuichiro Yamaguchi ${ }^{1}$, Takeshi Kawakami ${ }^{1}$, Takuya Wada ${ }^{1,2}$, Kimihiro \\ Igarashi ${ }^{1}$, Yoshihiro Kishida ${ }^{1}$, Yusuke Kinugasa ${ }^{3}$, Noboru Kawata ${ }^{1}$, Masaki Tanaka ${ }^{1}$, Naomi Kakushima ${ }^{1}$, Kohei \\ Takizawa $^{1}$, Hirotoshi Ishiwatari ${ }^{1}$, Hiroyuki Matsubayashi' ${ }^{1}$, Hiroyuki Ono ${ }^{1}$
}

Institutions

1 Division of Endoscopy, Shizuoka Cancer Center, Shizuoka, Japan

2 Department of Gastroenterology, Kitasato University School of Medicine, Kanagawa, Japan

3 Division of Colorectal Surgery, Shizuoka Cancer Center, Shizuoka, Japan

submitted 8.10 .2016

accepted after revision 2.3.2017

Bibliography

DOI https://doi.org/10.1055/s-0043-106182 |

Endoscopy International Open 2017; 05: E513-E517

(c) Georg Thieme Verlag KG Stuttgart · New York

ISSN 2364-3722

Corresponding author

Kenichiro Imai, Division of Endoscopy, Shizuoka Cancer

Center, 1007 Shimonagakubo, Nagaizumi, Suntogun,

Shizuoka, 411-8777, Japan

Fax: +81-55-989-5692

k.imai1977@gmail.com

\section{ABSTRACT}

Background and study aims In colorectal cancer surgery, inadvertent deep injections during endoscopic tattooing can cause India ink leakage into the peritoneum, leading to complications or to poor visualization of the surgical plane. This ex vivo animal study compared the use of novel shorter, minimum caliber needles versus conventional injection needles for endoscopic tattooing.

Animals and methods Four endoscopists used the novel needles and conventional needles to make ten endoscopic tattoos (five tattoos/needle type/endoscopist) in harvested porcine rectum using a saline test-injection method. India ink leakage and the success of the tattoo (i.e. visible, $<40 \mathrm{~mm}$, plus no India ink leakage) were compared.

Results India ink leakage was observed for $30 \%(6 / 20)$ of the conventional needle tattoos but for none of the novel needle tattoos $(P=0.02)$. Tattoos created using the novel needles were more successful than those made with the conventional needles: 18/20 (90\%) vs. $11 / 20$ (55\%); $P=0.01$.

Conclusions The use of novel shorter minimum caliber needles may be safe and effective for endoscopic tattooing for preoperative localization prior to colorectal cancer surgery.

\section{Introduction}

Laparoscopic surgery for colorectal cancer has become increasingly common due to recent comparisons of survival and recurrence after laparoscopic versus open surgery [1]. Accurate tumor localization is needed prior to laparoscopic surgery for colorectal cancers, especially for impalpable lesions [2]. For decades, endoscopic tattooing using India ink has been considered a safe and effective method for preoperative localization [3]. However, when India ink is injected deeply into the peritoneum, the resulting decreased tattoo visibility can hinder localization and India ink leakage can lead to harmful complications such as abscess formation or peritonitis $[4,5]$. Moreover, ink leakage itself is reported to increase technical difficulties in the subsequent surgery due to poor visualization of the surgical plane [6]. The saline test-injection method decreases the risks of deep injection [7]; however, even with this technique, the reported rates of India ink leakage of $9 \%-14 \%$ remain unacceptably high $[3,8,9]$. Moreover, the "dead space" of the injector's catheter increases the amount of injected saline, which can lead to a tattoo that is larger than intended and to excessive dilution of the India ink [10]. This may present a barrier to using endoscopic tattooing prior to sphincter-saving super-low anterior resections (anastomosis located within $20 \mathrm{~mm}$ of the dentate line). The aim of this ex vivo animal study was to compare the performance of endoscopic tattooing using newly devel- 


\begin{tabular}{llll}
$1.9 \mathrm{~mm}$ & Novel shorter needle \\
$2.6 \mathrm{~mm}$ & & & \\
& & & \\
Needle type & Catheter length & Catheter caliber & Catheter volume \\
$\begin{array}{l}\text { Novel shorter } \\
\text { needle }\end{array}$ & $2200 \mathrm{~mm}$ & $1.9 \mathrm{~mm}$ & $0.3 \mathrm{~mL}$ \\
$\begin{array}{l}\text { Conventional } \\
\text { needle }\end{array}$ & $2600 \mathrm{~mm}$ & $2.6 \mathrm{~mm}$ & $3 \mathrm{~mL}$ \\
\hline
\end{tabular}

- Fig. 1 Product specification of the novel shorter minimum caliber tattoo needle and the conventional needle.

oped, shorter-length and minimum caliber needles versus conventional injection needles.

\section{Animals and methods}

\section{Novel tattoo needle}

A novel tattoo needle was developed in collaboration with the TOP Corporation, Tokyo, Japan ( $\triangleright$ Fig. 1). The novel needle was modified as follows: (1) To reduce the risk of penetration of the thin colorectal wall, a shorter $(2.5-\mathrm{mm})$ needle was used; and (2) To reduce the injector catheter volume, a smaller caliber $(1.9-\mathrm{mm})$ injector catheter was used. These modifications reduced the volume of the injector catheter's lumen to $0.3 \mathrm{~mL}$, which should help reduce excessive dilution and the unintended expansion of the India ink due to the "boost" injection of saline within the injector catheter. The novel needle can be provided at similar costs to the conventional injection needle.

\section{Ex vivo porcine model}

Harvested porcine rectum that is commercially available for human consumption was used in this study for ethical reasons. For the ex vivo porcine model, the porcine rectum was irrigated with water to remove the contents. After one end of the rectum was closed using forceps, the absence of leakage was confirmed by filling it with water. An endoscopic overtube (TOP, Tokyo, Japan) was connected to one end of the rectum for insertion of the endoscope, and the rectum was fixed to a square plastic frame ( $\triangleright$ Fig. 2 ).

\section{Endoscopic tattooing}

Endoscopic tattooing was performed by 4 endoscopists (K. I., K.H., T.W., and T.K.). Two were board-certificated endoscopists and members of the Japan Gastroenterological Endoscopy Society who had performed over 100 colonoscopic tattoo procedures. The other 2 were trainees in our institutional residency program who had performed fewer than 20 endoscopic tattoo procedures. A PCF-Q260 colonoscope (Olympus Optical Co., Ltd., Tokyo, Japan) and a disposable, 1-mL syringe (Nipro Corporation, Osaka, Japan) were used for the injections ( $\bullet$ Video 1 ). Each endoscopist performed 10 tattoos at the 3, 9, and

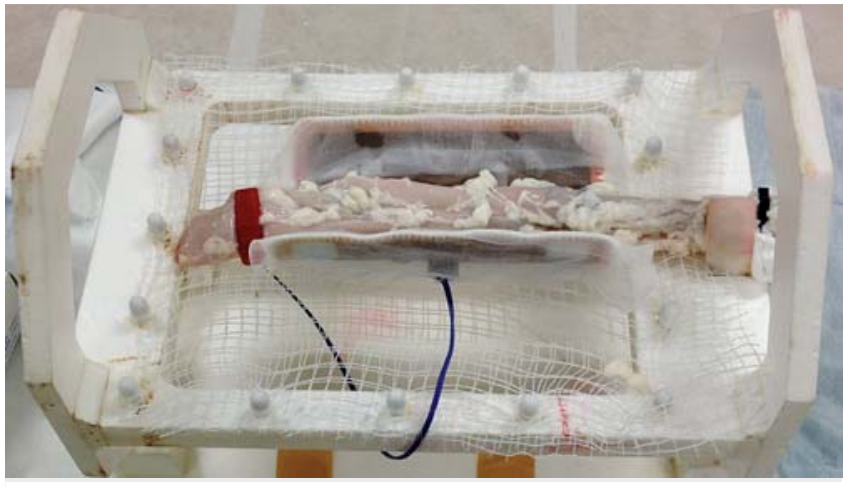

Fig. 2 The ex-vivo harvested porcine model.

12 o'clock positions of the colon model wall using a conventional needle (needle length, $4 \mathrm{~mm} ; \mathrm{n}=5$ ) or the novel short needle (needle length, $2.5 \mathrm{~mm} ; \mathrm{n}=5$ ). For the comparison experiment, each type of needle was handed to the endoscopist in a blinded manner with the needle lumen filled with saline solution. Endoscopic tattooing was performed using a saline test-injection method [7]. First, a submucosal cushion was created by an initial "test" submucosal injection of saline solution. Once the submucosal cushion began to form, the saline solution injection was stopped. Keeping the needle tip in the submucosa, the saline syringe was exchanged for the India ink syringe, and a fixed dose of India ink $(0.1 \mathrm{~mL})$ was injected. The India ink syringe was then exchanged for the saline syringe, and the "boost" saline was injected until submucosal bleb has changed black and become larger than $10 \mathrm{~mm}$.

\section{Endoscopic tattoo evaluation}

We compared the tattoos created using a conventional needle versus the novel needle by noting or measuring the following: the appearance of the tattoo from the serosa, the presence of India ink leakage, the major size of the tattoo axis as visualized

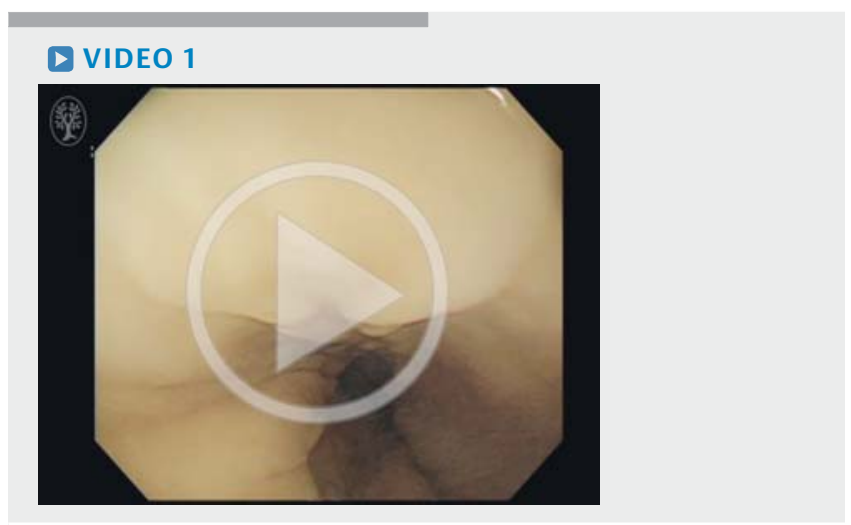

- Video 1: Endoscopic tattooing in a porcine model. The use of thin syringes, namely a $1-\mathrm{mL}$ syringe for India ink and a $5-\mathrm{mL}$ syringe for saline solution, is useful for reducing the pressure during the endoscopic tattooing procedure.

Online content viewable at: https://www.thieme-connect.com/ DOI/DOI?10.1055/s-0043-106182 


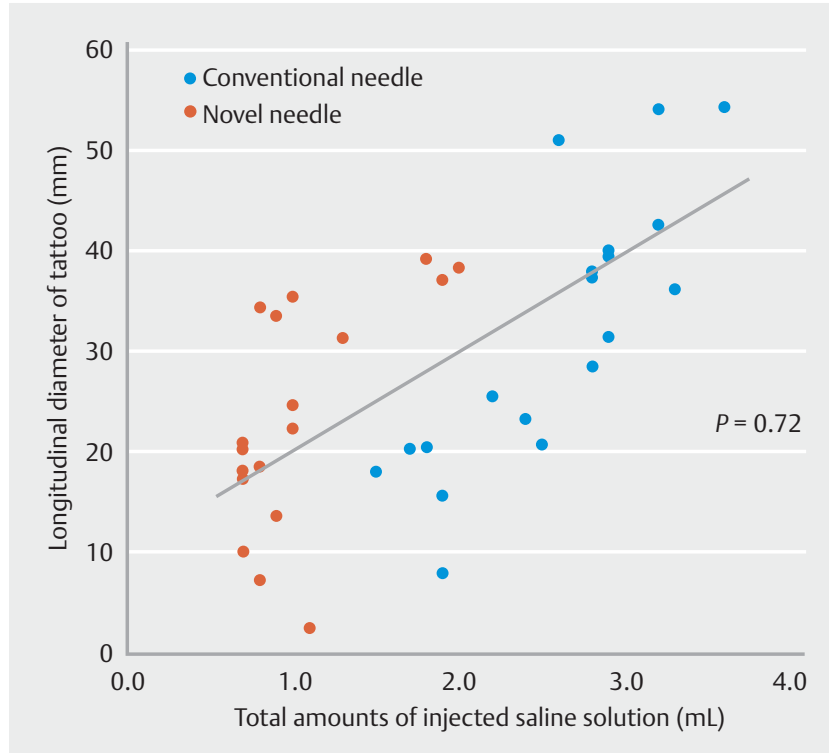

Fig. 3 Association between the maximum tattoo diameter and the total volume of injected saline solution. There was a significant linear association between the maximum tattoo diameter and the total volume of injected saline solution (Spearman's $\rho=0.72$, $P<0.0001)$.

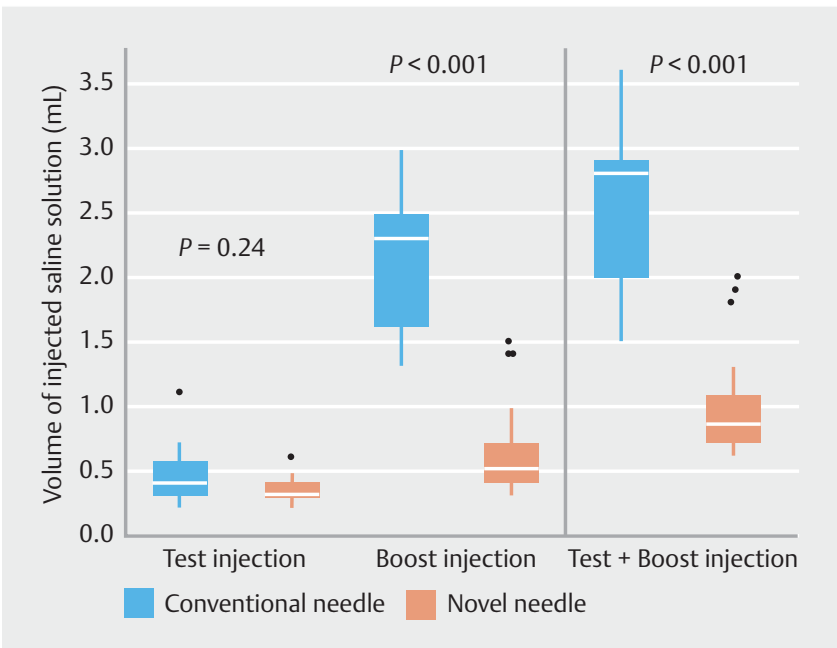

- Fig. 4 The volume of injected saline solution in the "test," "boost," and "test and boost" injections using the two kinds of needles. The blocks represent the $25^{\text {th }}$ to $75^{\text {th }}$ percentiles (blue blocks show data for conventional needles, and red blocks show data for novel needles). Black dots indicate the outliers, bars indicate the interquartile ranges $\left(5^{\text {th }}\right.$ to $95^{\text {th }}$ percentiles), and the white lines in the middle of the main blocks shows the median value $(P<0.0001$ for conventional needles versus novel needles for the boost injection).

from the serosa, the saline volume injection (test plus boost injections), and the success of the localized tattoo, where "success" meant that the tattoo was visible, smaller than $40 \mathrm{~mm}$, and had no India ink leakage. We compared India ink leakage rates and the volume of injected saline solution among endos- copists (Operator A and B; experienced endoscopists, Operator $\mathrm{C}$ and $\mathrm{D}$; less-experienced endoscopists).

\section{Statistics}

The Mann-Whitney $\mathrm{U}$ test was used to compare continuous variables, and the chi-squared test or Fisher's exact test, as appropriate, was used to compare categorical variables. All tests were two-tailed. A $P$ value $<0.05$ was considered to be statistically significant (JMP software, version 11.0; SAS Institute Inc., Cary, NC, USA).

\section{Results}

A total of $90 \%(18 / 20)$ of the tattoos created using the novel needle were visible from the serosa, whereas $100 \%(20 / 20)$ of the tattoos created using the conventional needle were visible $(P=0.49)$. No India ink leakage was observed for the novel needle tattoos $(0 / 20)$, but $30 \%(6 / 20)$ of the conventional needle tattoos showed leakage $(P=0.02)$. The median diameter of the visible tattoos was significantly smaller for the novel needle group than for the conventional needle group $(21.6 \mathrm{~mm}$ vs. $33.8 \mathrm{~mm}, P=0.04)$. There were significantly more successful localized tattoos (i.e. visible, $<40 \mathrm{~mm}$, and no India ink leakage) in the novel needle group versus the conventional needle group: $18 / 20(90 \%)$ vs. $11 / 20(55 \%)(P=0.01)$. There was a significant positive correlation between tattoo diameter and the total amount of injected saline solution $(P=0.72, P<0.001$; - Fig. 3). The median amount of injected saline solution for both the "test and boost" and the "boost" injection was significantly lower in the novel needle group than in the conventional group (total: $1 \mathrm{~mL}$ vs. $3 \mathrm{~mL}, \Delta=2.0 \mathrm{~mL}, P<0.001$; boost: $0.5 \mathrm{~mL}$ vs. $2.3 \mathrm{~mL}, \Delta=1.8 \mathrm{~mL}, P<0.001$, $>$ Fig. 4 ). There was no significant difference in terms of the volume of the injected saline solution or the tattoo diameter between the trainee endoscopists and the expert endoscopists. In comparison of endoscopists, India ink leakage occurred at various rates in each of endoscopists with the use of the conventional needle ( $\bullet$ Fig.5). In contrast, no India ink leakage was observed in the novel needle. In regard to the volume of injected saline solution, lower volume of "boost" injection was available by the use of the novel needle. The mean amounts of the "boost" injection were less than $0.5 \mathrm{~mL}$ in Endoscopist A, B, and C, whereas Endoscopist D injected more than $1.0 \mathrm{~mL}$ of saline.

\section{Discussion}

This experimental study revealed that this novel smaller needle had two advantages for endoscopic tattooing. First, the shorter length of the needle may minimize the risk of leakage into the peritoneum. Generally, when the needle can be inserted at an oblique angle to the bowel wall, penetration of the serosa is rare. However, this may depend on the endoscopist's skills. In this study, no leakage was observed in the novel needle group even when trainees performed the injections. This suggests that the novel needle may be useful for preventing too-deep injections regardless of the endoscopist's skills or experience. Second, the smaller caliber of the novel needle allowed the 


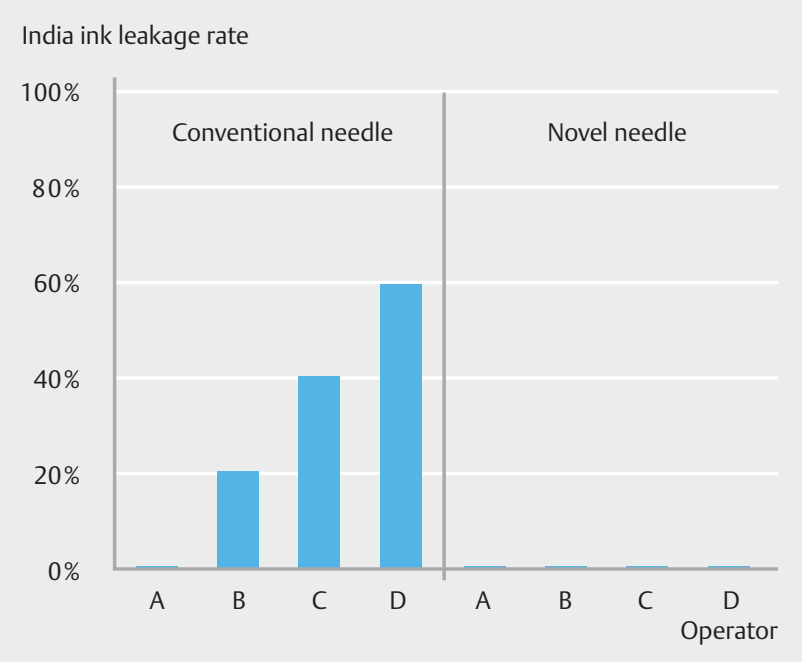

- Fig. 5 India ink leakage rates in each of endoscopists using conventional and the novel needle. In the conventional needle, India ink leakage occurred at various rates in each of endoscopists. In contrast, no India ink leakage was observed in the novel needle. endoscopists to create more localized tattoos. We found that greater saline injection volumes were associated with larger tattoo diameters ( $\triangleright$ Fig. 3 ). The total volume of the injected saline solution was significantly lower in the novel needle group than in the conventional needle group, mainly due to the smal-

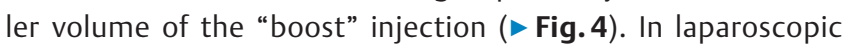
surgery for rectal cancer, the minimum distal surgical margin is considered $2 \mathrm{~cm}$ because lymph node metastasis rarely occurs more distally than $2 \mathrm{~cm}[11-13]$. When preoperative endoscopic tattooing is performed distal to the rectal cancer and the diameter of the tattoo is $>4 \mathrm{~cm}$ due to incidental India ink spreading, the resection line should be moved distally to near the anus. This may increase the risk of colostomy by involvement of the anastomosis to the anus. Considering the decrease in quality of life after colostomy, it is appropriate to aim for a tattoo that is $<40 \mathrm{~mm}$ in diameter for preoperative localization of rectal cancer.

Endoscopic tattooing may be affect endoscopist's skills. In - Fig. 6, endoscopists showed different India ink leakage rates in the conventional needle group. On the other hand, no India ink leakage occurred by each of endoscopist in novel needle
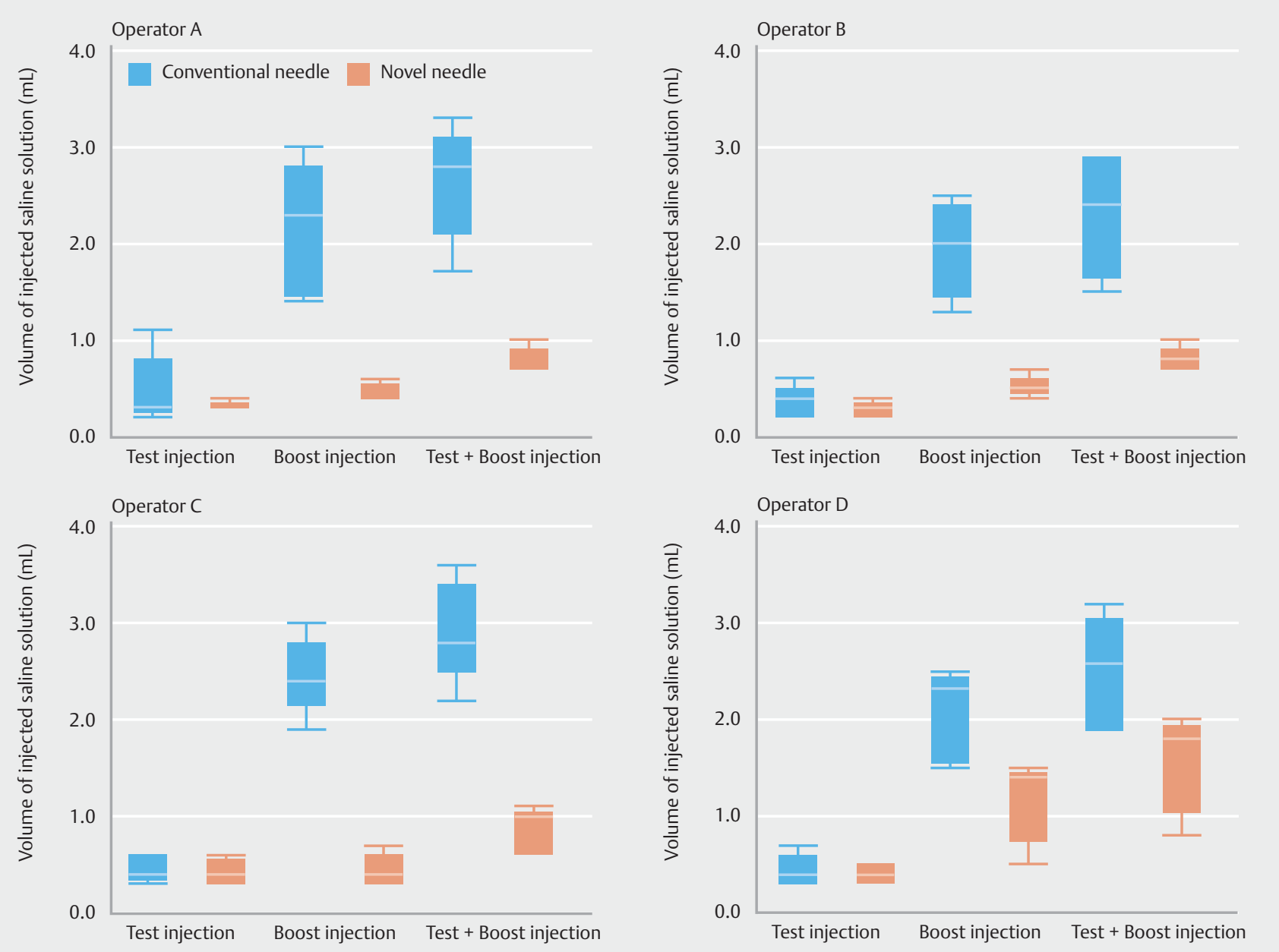

Fig. 6 The volume of injected saline solution in the "test," "boost," and "test and boost" injections using the two kinds of needles by endoscopists. 
group. This may imply that the novel needle enables successful submucosal injections regardless of endoscopist's experiences or skills.

We presented volumes of saline solution in "Test", "Boost", and "Test and Boost" by endoscopists in ₹ Fig. 6. Results in Operator $A, B$, and $C$ were similar, whereas Operator D injected more amounts of saline solution in the "Boost" injection. This may indicate that some of less-experienced endoscopists don't recognize appropriate amounts of "boost" injection for a visible, localized tattoo because appropriate amounts of "boost" injection have not been introduced.

There are no guidelines governing the injection number, location, volume, or the direction of the tattoo, so these parameters continue to be debated. Some studies have suggested the use of four-quadrant tattoos or two-opposite side tattoos at the distal side of the lesion $[14,15]$. However, considering the increased risk of ink leakage due to the increasing number of tattoo injections, it is important to develop a tattooing technique that ensures that the tattoo is visible while minimizing the number of injections. The results of this study indicate that a single tattoo injection using the saline test-injection method and this novel needle could provide tattoos that are visible $90 \%$ of the time, are localized, and that do not have ink leakage.

Further consideration is other potential applications of the novel needle. Actually, it is an attractive idea to use the needle for endoscopic mucosal resection (EMR) or endoscopic submucosal dissection (ESD). Currently, we consider it is inconvenient to use the needle in EMR or ESD. Its minimal caliber requires a higher pressure for pushing a syringe for injection of India ink and saline. The injection of high viscous solutions via the novel needle by manual pressing may be difficult. Mechanical injection system will enable injection of high viscous solutions with this novel needle. Notably, short length of needle is effective for appropriate injection directly into the submucosal layer. Therefore, short-length needle of $2.5 \mathrm{~mm}$ may be an applicable design for EMR or ESD procedures.

This study was an ex vivo animal study. Given different anatomical features such as thickness of the submucosa between human and this animal model, a human study is necessary. Therefore, the safety and effectiveness of this tattooing technique must be confirmed in a human study. We therefore initiated a prospective feasibility study involving patients undergoing surgery for colorectal cancer.

\section{Conclusion}

To summarize, this newly developed shorter minimum caliber needle may be useful for endoscopic tattooing as it reduces India ink leakage while providing a more localized mark.
Competing interests

None

\section{References}

[1] Bonjer HJ, Deijen CL, Abis GA et al. A Randomized trial of laparoscopic versus open surgery for rectal cancer. N Engl J Med 2015; 372: 1324 1332

[2] Vaziri K, Choxi SC, Orkin BA. Accuracy of colonoscopic localization. Surg Endosc 2010; 24: 2502-2505

[3] Askin MP, Waye JD, Fiedler L et al. Tattoo of colonic neoplasms in 113 patients with a new sterile carbon compound. Gastrointest Endosc 2002; 56: $339-342$

[4] Alba LM, Pandya PK, Clarkston WK. Rectus muscle abscess associated with endoscopic tattooing of the colon with India ink. Gastrointest Endosc 2000; 52: 557- 558

[5] Gopal DV, Morava-Protzner I, Miller HA et al. Idiopathic inflammatory bowel disease associated with colonic tattooing with india ink preparation-case report and review of literature. Gastrointest Endosc 1999; 49: 636-639

[6] Imai K, Hotta K, Kinugasa Y. Difficult laparoscopic total mesorectal excision after preoperative colonoscopic tattooing. Digestive Endoscopy 2016; 28: $102-102$

[7] Fu KI, Fujii T, Kato S et al. A new endoscopic tattooing technique for identifying the location of colonic lesions during laparoscopic surgery: a comparison with the conventional technique. Endoscopy 2001; 33: 687-691

[8] Park JW, Sohn DK, Hong CW et al. The usefulness of preoperative colonoscopic tattooing using a saline test injection method with prepackaged sterile India ink for localization in laparoscopic colorectal surgery. Surg Endosc 2008; 22: $501-505$

[9] Arteaga-Gonzalez I, Martin-Malagon A, Fernandez EM et al. The use of preoperative endoscopic tattooing in laparoscopic colorectal cancer surgery for endoscopically advanced tumors: a prospective comparative clinical study. World J Surg 2006; 30: 605-611

[10] Moss A, Bourke M], Pathmanathan N. Safety of colonic tattoo with sterile carbon particle suspension: a proposed guideline with illustrative cases. Gastrointest Endosc 2011; 74: 214-218

[11] Shimada Y, Takii Y, Maruyama $S$ et al. Intramural and mesorectal distal spread detected by whole-mount sections in the determination of optimal distal resection margin in patients undergoing surgery for rectosigmoid or rectal cancer without preoperative therapy. Dis Col Rectum 2011; 54: $1510-1520$

[12] Ono C, Ono K, Yoshinaga M et al. Discontinuous Rectal Cancer Spread in the Mesorectum and the Optimal Distal Clearance Margin in Situ. Dis Col Rectum 2002; 45: $744-749$

[13] Shirouzu K, Shirouzu H, Isomoto T et al. Distal spread of rectal cancer and optimal distal margin of resection for sphincter-preserving surgery. Cancer 1995; 76: 388-392

[14] Hyman N, Waye JD. Endoscopic four quadrant tattoo for the identification of colonic lesions at surgery. Gastrointest Endosc 1991; 37: $56-58$

[15] Moss A. Colonic tattooing: the revival of a black art? Gastrointest Endosc 2012; 76: $801-803$ 